\title{
ENURESIS- A NEW COMORBIDITY OF CHILDHOOD OBESITY?
}

Y. Weintraub ${ }^{1,2}$, S. Zinger ${ }^{3}$, S. Chacham ${ }^{3}$, D. Alexander ${ }^{3}$, G. Menuchin ${ }^{4}$, R. Lubezky ${ }^{5}$, O. PinnhasHamiel $^{2,4}$

${ }^{I}$ Sheba Medical Center, Safra Children's Hospital, Ramat Gan, ${ }^{2}$ Sackler School of Medicine, Tel Aviv University, ${ }^{3}$ Maccabi Health Services, Tel Aviv, ${ }^{4}$ Pediatric Endocrinology Unit, Sheba Medical Center, Safra Children's Hospital, Ramat Gan, ${ }^{5}$ Soraski Medical Center, Dana Children's Hospital, Tel Aviv, Israel

Introduction: Obese children are at increased risk to develop severe comorbidities, including: Type 2 Diabetes Mellitus, Obstructive Sleep Apnea (OSA), Attention Deficit/Hyperactivity Disorder (ADHD) and variable psychopathology.

Enuresis prevalence is increased in these specific medical conditions.

This study aims to assess the association between obesity and enuresis in children and adolescents.

Methods: A prospective study was performed among 344 subjects, aged 7-18 years. A questionnaire evaluated nocturnal enuresis, comorbidites and sociodemographic data. 281 subjects completed the questionnaires, 158 were normal weight, 37 overweight $(85 \leq \mathrm{BMI} \leq 95$ percentiles, Body mass index(BMI), for age and gender) and 86 obese (BMI $\geq 95$ th percentile).

Outcome measures: height, weight, BMI and BMI-Z-score(BMIZ), prevalence of enuresis, association between enuresis and gender, family history, voiding dysfunction, ADHD, OSA and sociodemographic variables.

Results: Enuresis was present in $14(8.8 \%)$ of normal weight subjects, $6(16 \%)$ of overweight and $26(30 \%)$ of obese youth. A significant independent risk for enuresis was present in obese children ( $\mathrm{OR}=6.5,95 \%$ $\mathrm{CI}(2.67-15.78))$ compared to normal weight $[\mathrm{P}<0.0001]$. One unit increase in BMIZ, increases the risk for enuresis by $\mathrm{OR}$ of $2.14, \mathrm{P}=0.00008,95 \% \mathrm{CI}(1.46-3.12)$.

Male gender [OR-2.84, $\mathrm{P}=0.028,95 \% \mathrm{CI}(1.10-5.58)]$,first degree relative with current/past enuresis [OR4.24, $\mathrm{P}=0.003,95 \% \mathrm{CI}(1.62-11.08)]$, voiding dysfunction symptoms [OR-3.067, $\mathrm{P}=0.041,95 \% \mathrm{CI}(1.05-9.00)$ ] and $\mathrm{ADHD}[\mathrm{OR}-2.31, \mathrm{P}=0.051,95 \% \mathrm{CI}(0.99-5.34)]$ increase the subjects risk to suffer from enuresis .

Conclusions: Enuresis in more prevalent among obese children. The joint risk factors support the hypothesis of a relation between the two entities. Due to this association, enuresis should be clarified during the primary workup of every obese child and adolescent. 